\title{
Smart Microscopy for Multi-Scale Developmental Biology in Real-Time
}

\author{
Jan Huisken ${ }^{1,2}$ \\ 1. Dept. of Medical Engineering, Morgridge Institute for Research, Madison, WI, USA \\ 2. Dept. of Biomedical Engineering, University of Wisconsin, Madison, WI, USA
}

Our understanding of developmental processes in biology relies crucially on their observation in living organisms. Morphogenesis is a dynamic process on many scales, governed by the shape changes, movements, divisions and interactions of its basic building blocks, the cells. For an understanding of the underlying biological mechanisms it is therefore desirable to simultaneously capture the dynamic behavior of cells and their interactions across the entire embryo over long periods of time.

Imaging a single embryo with high spatial and temporal resolution will reveal details of the developmental processes in the specific embryo under observation. However, the information from each individual sample needs to be compared to a large ensemble to quantify the variability and the significance of the findings. It is indispensable to learn about nature's variability first, before any conclusion can be drawn about, e.g. the robustness of a mutant phenotype, the adaptation of an organism to environmental constrains, or the effect of a drug. A major requirement for future quantitative developmental biology is therefore the ability to image and efficiently analyze a large number of embryos with sufficient spatial and temporal resolution across their entire extent without compromising their health.

Unfortunately, currently no microscopy platform can provide all of this in a single instrument. It is therefore a major goal in biological imaging to develop a device that delivers 3D data across time for many samples while, at the same time, delivering the tools to analyze and compare the data rigorously for a statistically relevant result. This would finally allow us to address fundamental questions such as how conserved embryonic development is and precisely how one embryo differs from another.

Fluorescence in vivo microscopy is the tool of choice for imaging cellular structures in biological samples. In particular, confocal microscopy and two-photon microscopy are widely used for imaging tissue sections and small regions in intact organisms. However, these techniques are not suitable for in toto imaging of millimeter-sized embryos due to these microscopes' low speed and high photo-toxicity. These limitations are also not addressed by a number of recently presented techniques that provide resolution beyond the diffraction limit (structured illumination microscopy, STED, PALM, etc.).

We introduced light sheet microscopy to the field of biological imaging in 2004. Since then Selective Plane Illumination Microscopy (SPIM) [1] has been shown to be a valuable technology that performs especially well in samples that are too large for conventional techniques [2]. In SPIM, instantaneous optical sectioning is achieved by illuminating the sample with a sheet of light and generating fluorescence in a thin slice, which is then imaged with a fast camera. Millimeter-sized specimens can be reconstructed by rotating them and imaging them from different sides (multi-view imaging) [3]. Phototoxicity is negligible even at high acquisition rates, making SPIM the ideal platform for long time-lapse experiments. 
To address this long-standing multi-disciplinary challenge, we are developing an entirely novel microcopy hard- and software platform (smart microscope) to systematically image and analyze embryos in real time [4]. We have built a fast and flexible multimodal light-sheet microscope (SPIM) with adaptive illumination and detection from multiple sides. A fundamentally new concept of this system is the ability to adaptively change the recording's spatial and temporal resolution during the experiment: The microscope learns to acquire only the data of interest. Using a high-throughput sample feeder, many samples can be automatically pumped through the microscope and imaged within seconds for largescale comparative developmental studies. Real-time processing will dramatically reduce the size of the data stream and thus, provide for the first time a platform to collect data from hundreds of samples.

\section{References:}

[1] J. Huisken, J. Swoger, F. Del Bene, J. Wittbrodt, E. H. K. Stelzer, Science 305, 1007 (2004).

[2] R.M. Power, J. Huisken, Nat Meth (2017, in press).

[3] B. Schmid et al., Nat Commun 4, 2207 (2013).

[4] N. Scherf, J. Huisken, Nat Biotechn 33, 815 (2015).

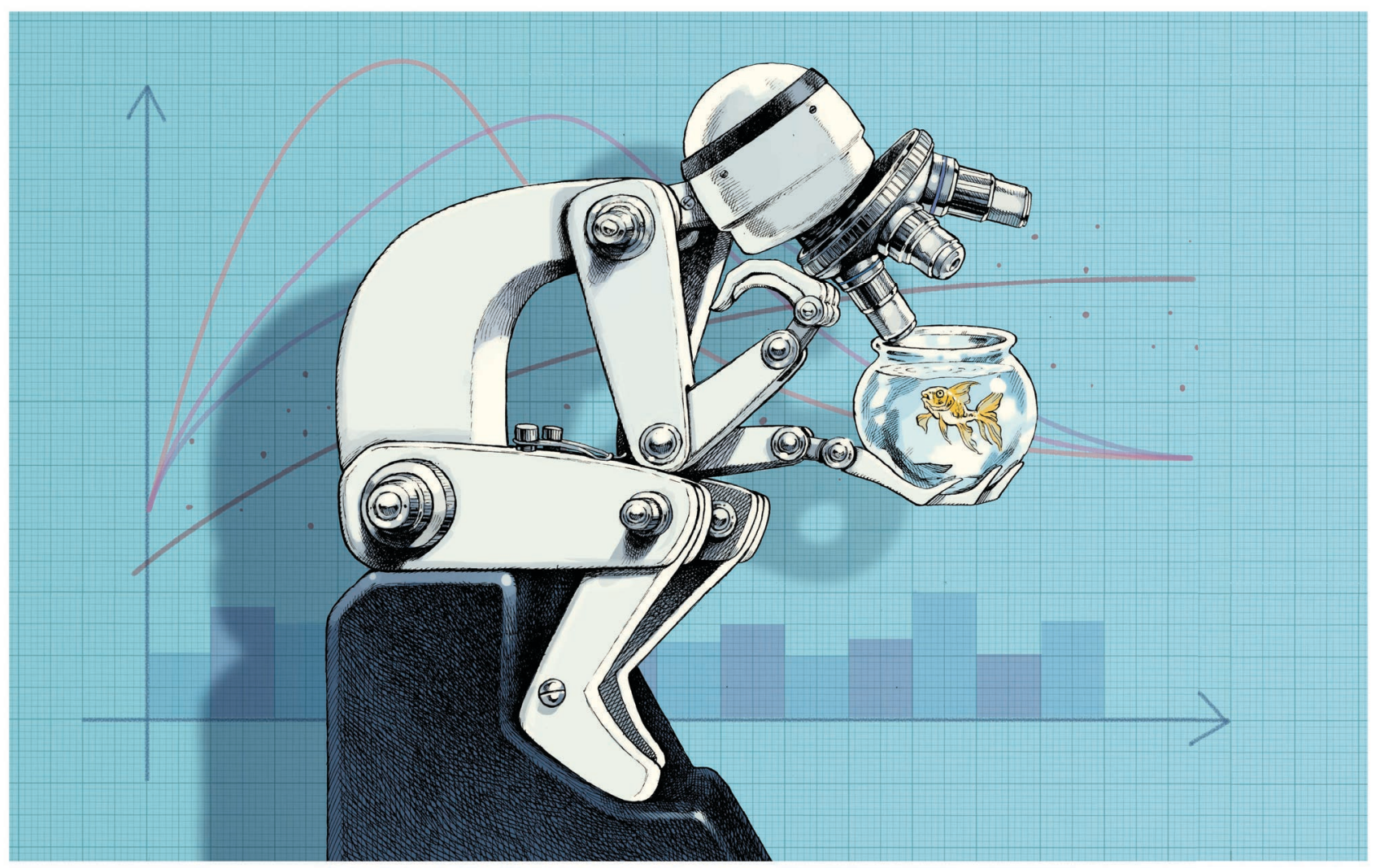

Figure 1. The smart microscope observes a freely swimming fish (by David Parkins, from [4]). 